Canad. Math. Bull. Vol. 20 (2), 1977

\title{
TAUBERIAN THEOREMS FOR STRONG AND ABSOLUTE BOREL-TYPE METHODS OF SUMMABILITY ${ }^{(1)}$
}

\author{
BY \\ D. BORWEIN AND E. SMET
}

1. Introduction. Suppose throughout that $s, a_{n}(n=0,1,2, \ldots)$ are arbitrary complex numbers, that $\alpha>0$ and $\beta$ is real and that $N$ is a non-negative integer such that $\alpha N+\beta \geq 1$. Let

$$
\begin{aligned}
s_{n} & =\sum_{\nu=0}^{n} a_{\nu}(n \geq 0), & s_{-1} & =0, \\
s_{\alpha, \beta}(z) & =\sum_{n=N}^{\infty} s_{n} \frac{z^{\alpha n+\beta-1}}{\Gamma(\alpha n+\beta)}, & a_{\alpha, \beta}(z) & =\sum_{n=N}^{\infty} a_{n} \frac{z^{\alpha n+\beta-1}}{\Gamma(\alpha n+\beta)}, \\
S_{\alpha, \beta}(z) & =\alpha e^{-z} s_{\alpha, \beta}(z), & A_{\alpha, \beta}(z) & =\alpha e^{-z} a_{\alpha, \beta}(z)
\end{aligned}
$$

where $z=x+i y$ is a complex variable and the power $z^{\gamma}$ is assumed to have its principal value.

Borel-type methods are defined as follows:

(a) Summability: If $S_{\alpha, \beta}(x)$ exists for all $x \geq 0$ and tends to $s$ as $x \rightarrow \infty$, we say that $s_{n} \rightarrow s(B, \alpha, \beta)$ or $\sum_{0}^{\infty} a_{n}=s(B, \alpha, \beta)$;

(b) Strong summability with index $p>0$ : If $S_{\alpha, \beta-1}(x)$ exists for all $x \geq 0$ and

$$
\int_{0}^{x} e^{t}\left|S_{\alpha, \beta-1}(t)-s\right|^{p} d t=o\left(e^{x}\right)
$$

we say that $s_{n} \rightarrow s[B, \alpha, \beta]_{p}$;

(c) Absolute summability: If $s_{n} \rightarrow s(B, \alpha, \beta)$ and $S_{\alpha, \beta}(x) \in B V_{x}[0, \infty)$, (2) we say that $s_{n} \rightarrow s|B, \alpha, \beta|$;

(d) Boundedness: If $S_{\alpha, \beta}(x)$ exists and is bounded on $[0, \infty)$, we say that $s_{n}=0(1)(B, \alpha, \beta)$;

(e) Strong boundedness with index $p>0$ : If $S_{\alpha, \beta-1}(x)$ exists for all $x \geq 0$ and

$$
\int_{0}^{x} e^{t}\left|S_{\alpha, \beta-1}(t)\right|^{p} d t=0\left(e^{x}\right)
$$

we say that $s_{n}=0(1)[B, \alpha, \beta]_{p}$.

Received by the editors January 13, 1976.

(1) This research has been supported by Grant A2983 of the National Research Council of Canada.

${ }^{(2)} f(x) \in B V_{x}[0, \infty)$ means that $f(x)$ is of bounded variation with respect to $x$ on $[0, \infty)$. 
The summability method $(B, 1,1)$ is the Borel exponential method B (see [7]). The $(B, \alpha, \beta)$ method is due to Borwein (see [2]) and the $[B, \alpha, \beta]_{p}$ and $|B, \alpha, \beta|$ methods are due to Borwein and Shawyer (see [4], [3] respectively). Strong Borel-type summability $[B, \alpha, \beta]$ (see [3]) is the $[B, \alpha, \beta]_{1}$ method.

The actual choice of the integer $N$ in the above definitions is clearly immaterial. We shall therefore tacitly assume whenever a finite number of methods, with $\alpha$ fixed and $\beta=\beta_{1}, \beta_{2}, \ldots, \beta_{k}$, are under consideration that $N$ is such that $\alpha N+\beta_{r} \geq 1(r=1,2, \ldots, k)$.

The following known result establishes a natural scale for these summability methods. (Theorem A(i) is [1, (II)]. Theorem $\mathrm{A}$ (ii) is [3, Theorem 9] when $p=1$ and part of $\left[4\right.$, Theorem $9^{*}$ (ii)] when $p \geq 1$. Theorem $\mathrm{A}$ (iii) is $[8$, Lemma].)

Theorem A. Let $\beta>\mu$.

(i) If $s_{n} \rightarrow s(B, \alpha, \mu)$, then $s_{n} \rightarrow s(B, \alpha, \beta)$.

(ii) If $p \geq 1$ and $s_{n} \rightarrow s[B, \alpha, \mu]_{p}$, then $s_{n} \rightarrow s[B, \alpha, \beta]_{p}$.

(iii) If $s_{n} \rightarrow s|B, \alpha, \mu|$, then $s_{n} \rightarrow s|B, \alpha, \beta|$.

In [5] we established a number of tauberian theorems for the $(B, \alpha, \beta)$ method. In this paper we investigate all the corresponding results for the $[B, \alpha, \beta]_{p}$ method with $p \geq 1$ and either prove them or show, by means of counterexamples, that they are false. We also examine some of the corresponding results for the $|B, \alpha, \beta|$ method.

2. Preliminary results. We first state some known results.

LEMMA 1.

(i) If $p \geq 1$ and $s_{n} \rightarrow s[B, \alpha, \beta]_{p}$, then $a_{n} \rightarrow 0[B, \alpha, \beta]_{p}$.

(ii) If $s_{n} \rightarrow s|B, \alpha, \beta|$, then $a_{n} \rightarrow 0|B, \alpha, \beta|$.

LEMMA 2.

(i) If $p \geq 1$ and $s_{n} \rightarrow s[B, \alpha, \beta]_{p}$, then $s_{n} \rightarrow s(B, \alpha, \beta)$.

(ii) If $p>0$ and $s_{n} \rightarrow s(B, \alpha, \beta)$, then $s_{n} \rightarrow s[B, \alpha, \beta+1]_{p}$.

Lemma 1(i) is included in [3, Theorem 15] when $p=1$ and in [4, Theorem $\left.15^{*}\right]$ when $p>1$. Lemma 1 (ii) is included in [3, Theorem 14]. Lemma 2(i) is [3, Theorem 3] when $p=1$ while Lemma 2(i) follows from [4, Theorem $\left.3^{*}\right]$ and Theorem A(i) when $p>1$. Lemma 2(ii) is [4, Theorem $\left.5^{*}\right]$.

Wherever it occurs in the following lemmas, we suppose that $f(x)$ is bounded and Lebesgue measurable on every finite interval $[0, X]$ and we let $f_{\delta}(x)$ be defined by

where $\delta>0$.

$$
f_{\delta}(x)=\frac{1}{\Gamma(\delta)} \int_{0}^{x}(x-t)^{\delta-1} f(t) d t
$$


LEMMA 3. If $\delta>0$ and $\gamma>0$, then

LeMma 4.

$$
f_{\delta+\gamma}(x)=\frac{1}{\Gamma(\gamma)} \int_{0}^{x}(x-t)^{\gamma-1} f_{\delta}(t) d t
$$

(i) Let $f(x)=s_{\alpha, \beta}(x)$ and let $\delta>0$. Then $s_{\alpha, \beta+\delta}(x)=f_{\delta}(x)$.

(ii) $A_{\alpha, \beta}(x)=S_{\alpha, \beta}(x)-S_{\alpha, \beta+\alpha}(x)-\alpha e^{-x} S_{N-1}\left(x^{\alpha N+\beta-1} / \Gamma(\alpha N+\beta)\right)$.

Lemma 3 is a well-known result the proof of which is straightforward. Lemma 4(i) is [2, Lemma 2]. The proof of Lemma 4(ii) is also straightforward.

LeMmA 5. If $s_{n}=0(1)(B, \alpha, \beta)$, then $s_{n}=0(1)(B, \alpha, \beta+\delta)$ for every $\delta>0$.

Lemma 5 is [3, Theorem 8].

LEMMA 6. Let $p \geq 1$. If $s_{n}=0(1)[B, \alpha, \beta]_{p}$, then

(i) $s_{n}=0(1)(B, \alpha, \beta)$,

(ii) $s_{n}=0(1)[B, \alpha, \beta+\delta]_{p}$ where $0<\delta<1$, and

(iii) $s_{n}=0(1)[B, \alpha, \beta+\delta]_{r}$ where $r>0$ and $\delta \geq 1$.

Proof. (i) When $p=1$ the result is [3, Theorem 4]. Thus we suppose that $p>1$ and we let $1 / p+1 / q=1$. Using Hölder's inequality and Lemma 4(i), we have that

$$
\begin{aligned}
\left|S_{\alpha, \beta}(x)\right| & \leq e^{-x} \int_{0}^{x} e^{t}\left|S_{\alpha, \beta-1}(t)\right| d t \\
& \leq e^{-x}\left\{\int_{0}^{x} e^{t}\left|S_{\alpha, \beta-1}(t)\right|^{p} d t\right\}^{1 / p}\left\{\int_{0}^{x} e^{t} d t\right\}^{1 / q} \\
& \leq e^{-x}\left\{K e^{x}\right\}^{1 / p}\left\{e^{x}\right\}^{1 / q}=K^{1 / p}
\end{aligned}
$$

for some positive constant $K$ since $s_{n}=0(1)[B, \alpha, \beta]_{p}$.

(ii) When $p=1$ the result is included in [3, Theorem 10]. Thus we again suppose that $p>1$ and we let $1 / p+1 / q=1$. Furthermore, we let $f(x)=\alpha s_{\alpha, \beta-1}(x), L=2^{p} /\{\Gamma(\delta)\}^{p}$, and $M=\left[1 /\{\Gamma(\delta)\}^{p}\right] \int_{0}^{1} e^{(1-p) t}\left|f_{\delta}(t)\right|^{p} d t$. Then, using Lemma 4(i), Hölder's inequality, and part of the proof of (i), we have for $x \geq 1$ that

$$
\begin{aligned}
\int_{0}^{x} e^{t}\left|S_{\alpha, \beta+\delta-1}(t)\right|^{p} d t= & \frac{1}{\{\Gamma(\delta)\}^{p}} \int_{0}^{x} e^{(1-p) t}\left|\int_{0}^{t}(t-u)^{\delta-1} f(u) d u\right|^{p} d t \\
\leq & L \int_{1}^{x} e^{(1-p) t}\left\{\int_{0}^{t-1}|f(u)| d u\right\}^{p} d t \\
& +L \int_{1}^{x} e^{(1-p) t}\left\{\int_{t-1}^{t}(t-u)^{\delta-1}|f(u)|^{p} d u\right\} \\
& \times\left\{\int_{t-1}^{t}(t-u)^{\delta-1} d u\right\}^{p / q} d t+M
\end{aligned}
$$




$$
\begin{aligned}
\leq & L \int_{0}^{x} e^{(1-p) t}\left\{K^{1 / p} e^{t}\right\}^{p} d t \\
& +\frac{L}{\delta^{p / q}} \int_{1}^{x} e^{(1-p) t} d t \int_{t-1}^{t}(t-u)^{\delta-1}|f(u)|^{p} d u+M \\
\leq & L K e^{x}+\frac{L}{\delta^{p-1}} \int_{0}^{x}|f(u)|^{p} d u \int_{u}^{u+1} e^{(1-p) t}(t-u)^{\delta-1} d t+M \\
\leq & L K e^{x}+\frac{L}{\delta^{p}} \int_{0}^{x} e^{(1-p) u}|f(u)|^{p} d u+M \\
= & L K e^{x}+\frac{L}{\delta^{p}} \int_{0}^{x} e^{u}\left|S_{\alpha, \beta-1}(u)\right|^{p} d u+M \\
= & 0\left(e^{x}\right) \quad \text { since } s_{n}=0(1)[B, \alpha, \beta]_{p} .
\end{aligned}
$$

This establishes the desired result.

(iii) If $\delta \geq 1$, then

$$
\int_{0}^{x} e^{t}\left|S_{\alpha, \beta+\delta-1}(t)\right|^{r} d t \leq \int_{0}^{x} K^{r} e^{t} d t \leq K^{r} e^{x}
$$

for some positive constant $K$ by Lemma 6(i) and Lemma 5.

LEMMA 7. If

$$
e^{-x} \int_{0}^{x} f(t) d t=o(1)
$$

then

$$
e^{-x} \int_{0}^{x} f_{\delta}(t) d t=o(1)
$$

for every $\delta>0$.

The proof of Lemma 7 is essentially the same as the proof of [3, Lemma 5].

LEMMA 8. Let $p \geq 1$. If

$$
e^{-x} \int_{0}^{x} e^{(1-p) t}|f(t)|^{p} d t=0(1) \quad \text { and } \quad e^{-x} \int_{0}^{x} f(t) d t=o(1)
$$

then

(i) $e^{-x} \int_{0}^{x} e^{(1-p) t}\left|f_{\delta}(t)\right|^{p} d t=o(1)$ where $0<\delta<1$ and

(ii) $e^{-x} \int_{0}^{x} e^{(1-r) t}\left|f_{\delta}(t)\right|^{r} d t=o(1)$ where $r>0$ and $\delta \geq 1$.

Proof. (i) Let $\varepsilon>0$. By hypothesis, there exists a number $Y \geq 0$ such that

$$
\left|\int_{0}^{x} f(t) d t\right| \leq \varepsilon e^{x}
$$


for all $x \geq Y$. Let

$$
N(\varepsilon)=\sup _{0 \leq x \leq Y}\left|\int_{0}^{x} f(t) d t\right|<\infty .
$$

Now

$$
\begin{aligned}
\underset{x \rightarrow \infty}{\limsup e^{-x} \int_{0}^{x} e^{(1-p) t}} \mid & \left.f_{\delta}(t)\right|^{p} d t \\
& =\limsup _{x \rightarrow \infty} e^{-x} \int_{\varepsilon}^{x} e^{(1-p) t}\left|\frac{1}{\Gamma(\delta)} \int_{0}^{t}(t-u)^{\delta-1} f(u) d u\right|^{p} d t \\
& \leq \frac{2^{p}}{\{\Gamma(\delta)\}^{p}}\left\{\limsup _{x \rightarrow \infty} I_{1}+\underset{x \rightarrow \infty}{\lim \sup _{2}} I_{2}\right\} .
\end{aligned}
$$

where

and

$$
I_{1}=e^{-x} \int_{\varepsilon}^{x} e^{(1-p) t}\left|\int_{0}^{t-\varepsilon}(t-u)^{\delta-1} f(u) d u\right|^{p} d t
$$

$$
I_{2}=e^{-x} \int_{\varepsilon}^{x} e^{(1-p) t}\left|\int_{t-\varepsilon}^{t}(t-u)^{\delta-1} f(u) d u\right|^{p} d t
$$

But, using the Second Mean Value Theorem,

$$
\begin{aligned}
\limsup _{x \rightarrow \infty} I_{1} & =\limsup _{x \rightarrow \infty} e^{-x} \int_{\varepsilon}^{x} e^{(1-p) t}\left|\varepsilon^{\delta-1} \int_{\mu(t)}^{t-\varepsilon} f(u) d u\right|^{p} d t \\
& \leq 2^{p} \varepsilon^{(\delta-1) p} \limsup _{x \rightarrow \infty} e^{-x} \int_{\varepsilon}^{x} e^{(1-p) t}\left\{N(\varepsilon)+\varepsilon e^{t}\right\}^{p} d t \\
& \leq 2^{2 p} \varepsilon^{(\delta-1) p} \limsup _{x \rightarrow \infty} e^{-x} \int_{\varepsilon}^{x} e^{(1-p) t}\left\{(N(\varepsilon))^{p}+\varepsilon^{p} e^{p t}\right\} d t \\
& =2^{2 p} \varepsilon^{\delta p}
\end{aligned}
$$

since

$$
\left|\int_{\mu(t)}^{t-\varepsilon} f(u) d u\right| \leq 2 \sup _{0 \leq y \leq t-\varepsilon}\left|\int_{0}^{y} f(u) d u\right| \leq 2\left\{N(\varepsilon)+\varepsilon e^{t}\right\}
$$

and

$$
\lim _{x \rightarrow \infty} e^{-x} \int_{\varepsilon}^{x} e^{(1-p) t}\{N(\varepsilon)\}^{p} d t=0
$$

Also, by hypothesis there is a number $K \geq 0$ such that

$$
e^{-x} \int_{0}^{x} e^{(1-p) t}|f(t)|^{p} d t \leq K
$$


for all $x \geq 0$, and therefore, when $p=1$,

$$
\begin{aligned}
& \limsup _{x \rightarrow \infty} I_{2} \leq \limsup _{x \rightarrow \infty} e^{-x} \int_{\varepsilon}^{x} d t \int_{t-\varepsilon}^{t}(t-u)^{\delta-1}|f(u)| d u \\
& \leq \limsup _{x \rightarrow \infty} e^{-x} \int_{0}^{x}|f(u)| d u \int_{u}^{u+\varepsilon}(t-u)^{\delta-1} d t \\
& \leq K \frac{\varepsilon^{\delta}}{\delta}
\end{aligned}
$$

while, when $p>1$,

$$
\begin{aligned}
\limsup _{x \rightarrow \infty} I_{2} \leq & \limsup _{x \rightarrow \infty} e^{-x} \int_{\varepsilon}^{x} e^{(1-p) t}\left\{\int_{t-\varepsilon}^{t}(t-u)^{\delta-1}|f(u)|^{p} d u\right\} \\
& \times\left\{\int_{t-\varepsilon}^{t}(t-u)^{\delta-1} d u\right\}^{p-1} d t \\
= & \left\{\frac{\varepsilon^{\delta}}{\delta}\right\}^{p-1} \limsup _{x \rightarrow \infty} e^{-x} \int_{\varepsilon}^{x} e^{(1-p) t} d t \int_{t-\varepsilon}^{t}(t-u)^{\delta-1}|f(u)|^{p} d u \\
\leq & \left\{\frac{\varepsilon^{\delta}}{\delta}\right\}^{p-1} \limsup _{x \rightarrow \infty} e^{-x} \int_{0}^{x}|f(u)|^{p} d u \int_{u}^{u+\varepsilon}(t-u)^{\delta-1} e^{(1-p) t} d t \\
& \leq\left\{\frac{\varepsilon}{\delta}\right\}^{p} \limsup _{x \rightarrow \infty} e^{-x} \int_{0}^{x} e^{(1-p) u}|f(u)|^{p} d u \leq K\left\{\frac{\varepsilon^{\delta}}{\delta}\right\}^{p} .
\end{aligned}
$$

Thus for $p \geq 1$ we have that

$$
\limsup _{x \rightarrow \infty} e^{-x} \int_{0}^{x} e^{(1-p) t}\left|f_{\delta}(t)\right|^{p} d t \leq \frac{2^{p}\left(2^{2 p}+K \delta^{-p}\right)}{\{\Gamma(\delta)\}^{p}} \varepsilon^{\delta p}
$$

from which it follows that

$$
\limsup _{x \rightarrow \infty} e^{-x} \int_{0}^{x} e^{(1-p) t}\left|f_{\delta}(t)\right|^{p} d t=0
$$

since $\varepsilon$ is arbitrary. This establishes the desired result.

(ii) Since $e^{-x} f_{1}(x)=o(1)$ by hypothesis, we have, when $\delta=1+\mu$ where $\mu>0$, that

$$
e^{-x} f_{1+\mu}(x)=e^{-x} \int_{0}^{x} f_{\mu}(t) d t=o(1),
$$

using Lemma 3 and Lemma 7. Hence, for $\delta \geq 1$,

$$
\begin{aligned}
e^{-x} \int_{0}^{x} e^{(1-r) t}\left|f_{\delta}(t)\right|^{r} d t & =e^{-x} \int_{0}^{x} e^{t}\left|e^{-t} f_{\delta}(t)\right|^{r} d t \\
& =e^{-x} \int_{0}^{x} e^{t} o(1) d t \\
& =o(1) .
\end{aligned}
$$


If $b$ is a real number, we let

$$
H_{b}=\{z \mid \operatorname{Re} z \geq b\} \text {. }
$$

A function $g(z)$ is said to be of exponential type in $H_{b}$ if $g(z)$ is analytic in $H_{b}$ and if there are positive numbers $A, a$ such that $|g(z)| \leq A e^{a|z|}$ for all $z$ in $H_{b}$.

Lemma 9. If $g(z)$ is of exponential type in $H_{0}$ and if

$$
\int_{0}^{\infty}|g(x)|^{p} d x<\infty \quad(p>0)
$$

then

$$
\int_{0}^{\infty}\left|g^{\prime}(x)\right|^{p} d x<\infty
$$

Lemma 9 is due to Gaier [6, Theorem 2].

Lemma 10. If $g(z)$ is of exponential type in $H_{b}$ and $g(x) \in B V_{x}[b, \infty)$, then

$$
g^{(k)}(x) \in B V_{x}[b, \infty)
$$

for every non-negative integer $k$.

Proof. Suppose that $g^{(k)}(x) \in B V_{x}[b, \infty)$ where $k$ is a non-negative integer. Then

$$
\int_{0}^{\infty}\left|g^{(k+1)}(x+b+1)\right| d x<\infty
$$

and

$$
\begin{aligned}
\left|g^{(k+1)}(z+b+1)\right| & \leq \frac{(k+1) !}{2 \pi} \int_{0}^{2 \pi}\left|g\left(z+b+e^{i \theta}\right)\right| d \theta \\
& \leq(k+1) ! A e^{a(|z|+|b|+1)}
\end{aligned}
$$

for all $z$ in $H_{0}$ where $A, a$ are positive constants. Hence, by Lemma 9,

$$
\int_{0}^{\infty}\left|g^{(k+2)}(x+b+1)\right| d x=\int_{b+1}^{\infty}\left|g^{(k+2)}(x)\right| d x<\infty
$$

i.e. $g^{(k+1)}(x) \in B V_{x}[b+1, \infty)$. Since $g^{(k+1)}(x) \in B V_{x}[b, b+1]$, therefore $g^{(k+1)}(x) \in$ $B V_{x}[b, \infty)$. The desired result now follows by induction.

3. Tauberian theorems for strong Borel-type summability with index $p \geq 1$. We first show that the scale in Theorem A(ii) us proper. In [5] we showed that there is a sequence $\left\{s_{n}\right\}$ which tends to a limit $(B, \alpha, \beta)$ but does not tend to a limit $(B, \alpha, \beta-1)$. Hence, in view of Lemma 2 , there is a sequence $\left\{s_{n}\right\}$ which tends to a limit $[B, \alpha, \beta+1]_{p}$ for every $p>0$ but does not tend to a limit $[B, \alpha, \beta-1]_{p}$ for any $p \geq 1$. 
THEOREM 1. Let $p, r \geq 1$. If $s_{n} \rightarrow s[B, \alpha, \mu]_{p}$ and $a_{n} \rightarrow 0[B, \alpha, \beta]_{r}$, then $s_{n} \rightarrow$ $s[B, \alpha, \beta]_{r}$.

Proof. By Lemma 2(i), $s_{n} \rightarrow s(B, \alpha, \mu)$. The result now follows by [9, Theorem 3] and the note following [9, Theorem 3].

THEOREM 2. Let $p \geq 1$. If $s_{n} \rightarrow s[B, \alpha, \beta+\varepsilon]_{p}$ for some $\varepsilon>0$ and $s_{n}=$ $0(1)[B, \alpha, \beta]_{p}$, then $s_{n} \rightarrow s[B, \alpha, \beta+\delta]_{p}$ for every $\delta>0$.

Proof. We can suppose without loss of generality that $s=0$. Then $s_{n} \rightarrow 0(B, \alpha, \beta+\varepsilon)$ and $s_{n}=0(1)(B, \alpha, \beta)$ by Lemma 2(i) and Lemma 6(i). Hence $s_{n} \rightarrow 0(B, \alpha, \beta+\delta)$ by [5, Theorem 2] for $\delta>0$. Also $s_{n}=0(1)[B, \alpha, \beta+\delta]_{p}$ by Lemma 6 (ii) or (iii). Therefore, letting $f(x)=\alpha s_{\alpha, \beta+\delta-1}(x)$, we have that

$$
e^{-x} \int_{0}^{x} f(t) d t=S_{\alpha, \beta+\delta}(x)=o(1)
$$

and

$$
e^{-x} \int_{0}^{x} e^{(1-p) t}|f(t)|^{p} d t=e^{-x} \int_{0}^{x} e^{t}\left|S_{\alpha, \beta+\delta-1}(t)\right|^{p} d t=0(1)
$$

using Lemma 4(i), and consequently,

$$
\left.e^{-x} \int_{0}^{x} e^{t}\left|S_{\alpha, \beta+2 \delta-1}(t)_{\mid}^{\mid p} d t=e^{-x} \int_{0}^{x} e^{(1-p) t}\right| f_{\delta}(t)\right|^{p} d t=o(1)
$$

using Lemma 4(i) and Lemma 8, i.e. $s_{n} \rightarrow 0[B, \alpha, \beta+2 \delta]_{p}$. This establishes the desired result.

TheOREM $2^{*}$. Let $p \geq 1$. If $\sum_{0}^{\infty} a_{n}=s[B, \alpha, \beta+\varepsilon]_{p}$ for some $\varepsilon>0$ and $a_{n}=0(1)[B, \alpha, \beta]_{p}$, then $\sum_{0}^{\infty} a_{n}=s[B, \alpha, \beta+\delta]_{p}$ for every $\delta>0$.

Proof. By Lemma 1(i), $a_{n} \rightarrow 0[B, \alpha, \beta+\varepsilon]_{p}$ and thus, by Theorem $2, a_{n} \rightarrow$ $0[B, \alpha, \beta+\delta]_{p}$ for every $\delta>0$. The result now follows by Theorem 1 .

A real-valued function $g(x)$, with domain $[0, \infty)$, is slowly decreasing if for every $\varepsilon>0$ there exist positive numbers $X, \delta$ such that $g(x)-g(y)>-\varepsilon$ whenever $x \geq y \geq X$ and $x-y \leq \delta$. The following result is [5, Theorem 3]: If $s_{n} \rightarrow s(B, \alpha, \beta+\varepsilon)$ for some $\varepsilon>0$ and $S_{\alpha, \beta}(x)$ is slowly decreasing, then $s_{n} \rightarrow s(B, \alpha, \beta)$. We now show that there is no analogue to this result for the $[B, \alpha, \beta]_{p}$ method.

Let $\left\{s_{n}\right\}$ be the sequence defined by $\sum_{n=0}^{\infty} s_{n}\left(x^{n} / n !\right)=e^{x} \sin e^{x}$ (cf. [7, p. 183]). Then $S_{1,1}(x)=\sin e^{x}$ where we choose $N=0$. Thus, using Lemma 4(i),

$$
S_{1,2}(x)=e^{-x} \int_{0}^{x} e^{t} \sin e^{t} d t=e^{-x}\left(\cos 1-\cos e^{x}\right)=o(1)
$$


and therefore $s_{n} \rightarrow 0(B, 1,2)$. (In fact, by [5, Theorem 2], $s_{n} \rightarrow 0(B, 1,1+\delta)$ for every $\delta>0$.) Hence, by Lemma 2(ii), $s_{n} \rightarrow 0[B, 1,3]_{r}$, for every $r>0$. Furthermore,

$$
\begin{aligned}
e^{-x} \int_{0}^{x} e^{t}\left|S_{1,1}(t)-0\right|^{r} d t & =e^{-x} \int_{0}^{x} e^{t}\left|\sin e^{t}\right|^{r} d t \\
& =e^{-x} \int_{1}^{e^{x}}|\sin u|^{r} d u \rightarrow \frac{L(r)}{\pi}
\end{aligned}
$$

as $x \rightarrow \infty$ where $L(r)=\int_{0}^{\pi}|\sin u|^{r} d u$. Therefore $s_{n} \nrightarrow 0[B, 1,2]_{r}, s_{n} \rightarrow 0[B, 1,3]_{r}$ and both $e^{-x} \int_{0}^{x} e^{t} S_{1,1}(t) d t$ and $e^{-x} \int_{0}^{x} e^{t}\left|S_{1,1}(t)\right|^{r} d t$ are slowly decreasing (since they both tend to a limit as $x \rightarrow \infty$ ).

THEOREM 3. Let $p \geq 1$. If $s_{n} \rightarrow s[B, \alpha, \mu]_{p}$ and

(i) $s_{n} \geq-K$ for all $n \geq 0$, or

(ii) $a_{n} \geq-K$ for all $n \geq 0$, or

(iii) $S_{\alpha, \mu}(z)$ is of exponential type in $H_{\delta}$, or

(iv) $A_{\alpha, \mu}(z)$ is of exponential type in $H_{\delta}$, or

(v) $\left|a_{n}\right| \leq K^{n}$ for all $n \geq 0$,

where $K, \delta$ are positive constants, then

$$
s_{n} \rightarrow s[B, \alpha, \beta]_{r}
$$

for every $r>0$.

Proof. By Lemma 2(i), $s_{n} \rightarrow s(B, \alpha, \mu)$. Hence, by [5, Theorem 5, 5*, 6, 6*, or 7], $s_{n} \rightarrow s(B, \alpha, \beta-1)$. The result now follows by Lemma 2(ii).

4. Tauberian theorems for absolute Borel-type summability. We first show that the scale in Theorem $A($ iii) is proper in the sense that for each $\beta$ there is a sequence $\left\{s_{n}\right\}$ which is summable $|B, \alpha, \beta|$ but is not summable $|B, \alpha, \beta-1|$.

Choose an integer $m$ such that $\alpha m>1$ and let $P$ be the smallest integer such that $m P \geq N$. Let

$$
x^{P} e^{-x} \sin e^{x}=\sum_{n=P}^{\infty} b_{n} x^{n}
$$

and let

$$
s_{n}= \begin{cases}\Gamma(\alpha n+\beta) b_{k} & \text { if } n=m k \\ 0 & \text { otherwise }\end{cases}
$$

Then

$$
S_{\alpha, \beta}(x)=\alpha x^{\alpha m P+\beta-1} e^{-x} e^{-x^{\alpha m}} \sin e^{x^{\alpha m}}=o(1)
$$


and

$$
\begin{aligned}
S_{\alpha, \beta}^{\prime}(x)= & \alpha(\alpha m P+\beta-1) x^{\alpha m P+\beta-2} e^{-x} e^{-x^{\alpha m}} \sin e^{x^{\alpha m}} \\
& -\alpha x^{\alpha m P+\beta-1} e^{-x} e^{-x^{\alpha m}} \sin e^{x^{\alpha m}} \\
& -\alpha(\alpha m) x^{\alpha m P+\alpha m+\beta-2} e^{-x} e^{-x^{\alpha m}} \sin e^{x^{\alpha m}} \\
& +\alpha(\alpha m) x^{\alpha m P+\alpha m+\beta-2} e^{-x} \cos e^{x^{\alpha m}}
\end{aligned}
$$

so that $S_{\alpha, \beta}^{\prime}(x)=o(1)$ and $S_{\alpha, \beta}^{\prime}(x) \in L_{1}[0, \infty)$ since $\alpha m P+\beta-2 \geq \alpha N+\beta-2 \geq 0$ by our choice of $N$. Hence $s_{n} \rightarrow 0|B, \alpha, \beta|$. However

$$
S_{\alpha, \beta}^{\prime \prime}(x)=f(x)-\alpha(\alpha m)^{2} x^{\alpha m P+2 \alpha m+\beta-3} e^{-x} e^{x^{\alpha m}} \sin e^{x^{\alpha m}}
$$

where $f(x) \in L_{1}[0, \infty)$ and therefore $S_{\alpha, \beta}^{\prime \prime}(x) \notin L_{1}[0, \infty)$ since $\alpha m>1$. Thus, since

and

$$
S_{\alpha, \beta-1}(x)=S_{\alpha, \beta}(x)+S_{\alpha, \beta}^{\prime}(x)
$$

$$
S_{\alpha, \beta-1}^{\prime}(x)=S_{\alpha, \beta}^{\prime}(x)+S_{\alpha, \beta}^{\prime \prime}(x),
$$

we have that

$$
s_{n} \rightarrow 0(B, \alpha, \beta-1) \quad \text { but } \quad s_{n} \nrightarrow 0|B, \alpha, \beta-1|
$$

Theorem 4. If $s_{n} \rightarrow s|B, \alpha, \mu|$ and $a_{n} \rightarrow 0|B, \alpha, \beta|$, then $s_{n} \rightarrow s|B, \alpha, \beta|$.

Proof. By [5, Theorem 1], $s_{n} \rightarrow s(B, \alpha, \beta)$. Thus it remains only to show that $S_{\alpha, \beta}(x) \in B V_{x}[0, \infty)$. Let $k$ be a positive integer. Then, in view of Theorem $\mathrm{A}(\mathrm{iii}), A_{\alpha, \beta+(k-1) \alpha}(x) \in B V_{x}[0, \infty)$. Moreover, by Lemma 4(ii),

$$
S_{\alpha, \beta+(k-1) \alpha}(x)=A_{\alpha, \beta+(k-1) \alpha}(x)+S_{\alpha, \beta+k \alpha}(x)+\alpha e^{-x} S_{N-1} \frac{x^{\alpha N+\beta-1}}{\Gamma(\alpha N+\beta)}
$$

Therefore $S_{\alpha, \beta+(k-1) \alpha}(x) \in B V_{x}[0, \infty)$ if $S_{\alpha, \beta+k \alpha}(x) \in B V_{x}[0, \infty)$. Since, in view of Theorem A(iii), $S_{\alpha, \beta+k \alpha}(x) \in B V_{x}[0, \infty)$ when $\beta+k \alpha \geq \mu$, it readily follows that $S_{\alpha, \beta}(x) \in B V_{x}[0, \infty)$.

If $\left\{s_{n}\right\}$ is the sequence described in the paragraph preceding Theorem 3 , then, using Lemma 4(i),

$$
S_{1,3}(x)=e^{-x} \int_{0}^{x}\left(\cos 1-\cos e^{t}\right) d t
$$

and thus it is readily seen that $s_{n} \rightarrow 0|B, 1,3|$ and $s_{n} \rightarrow 0|B, 1,2|$. Hence there is also no immediate absolute summability analogue to [5, Theorem 3].

Our final results are extensions of a result due to Gaier (see [6]).

THEOREM 5. If $s_{n} \rightarrow s|B, \alpha, \mu|$ and $S_{\alpha, \mu}(z)$ is of exponential type in $H_{\delta}$ for some $\delta>0$, then $s_{n} \rightarrow s|B, \alpha, \beta|$. 
Proof. Let $k$ be a positive integer such that $\mu-k \leq \beta$. By [5, Theorem 6] we have that $s_{n} \rightarrow s(B, \alpha, \mu-k)$. Furthermore, since

$$
S_{\alpha, \mu-1}(z)=S_{\alpha, \mu}(z)+S_{\alpha, \mu}^{(1)}(z),
$$

it is readily seen that

$$
S_{\alpha, \mu-k}(z)=S_{\alpha, \mu}(z)+\sum_{j=1}^{k}\left(\begin{array}{l}
k \\
j
\end{array}\right) S_{\alpha, \mu}^{(j)}(z) .
$$

Since $S_{\alpha, \mu}(z)$ is of exponential type in $H_{\delta}$ and since $S_{\alpha, \mu}(x) \in B V_{x}[0, \infty)$ by hypothesis, we have, by Lemma 10 , that $S_{\alpha, \mu}^{(j)}(x) \in B V_{x}[\delta, \infty)$ for $j=1, \ldots, k$; also, since we choose $N$ so that $\alpha N+\mu-k \geq 1$, we have that $S_{\alpha, \mu}^{(j)}(x) \in B V_{x}[0, \delta]$ for $j=1, \ldots, k$. Therefore, $S_{\alpha, \mu}^{(j)}(x) \in B V_{x}[0, \infty)$ for $j=1, \ldots, k$ and, consequently, $S_{\alpha, \mu-k}(x) \in B V_{x}[0, \infty)$. Hence $s_{n} \rightarrow s|B, \alpha, \mu-k|$ and, by Theorem A(iii), $s_{n} \rightarrow s|B, \alpha, \beta|$.

THEOREM $5^{*}$. If $s_{n} \rightarrow s|B, \alpha, \mu|$ and $A_{\alpha, \mu}(z)$ is of exponential type in $H_{\delta}$ for some $\delta>0$, then $s_{n} \rightarrow s|B, \alpha, \beta|$.

Proof. By Lemma 1(ii), $a_{n} \rightarrow 0|B, \alpha, \mu|$ and thus, by Theorem 5, $a_{n} \rightarrow 0 \mid B, \alpha, \beta \|$. The result now follows by Theorem 4 .

THEOREM 6. If $s_{n} \rightarrow s|B, \alpha, \mu|$ and $\left|a_{n}\right| \leq K^{n}$ for all $n \geq 0$ where $K$ is a positive constant, then $s_{n} \rightarrow s|B, \alpha, \beta|$.

Proof. Since $\left|a_{n}\right| \leq K^{n}$ for all $n \geq 0$, we have that

$$
\left|A_{\alpha, \mu}(z)\right| \leq A e^{K^{1 / \alpha}|z|}
$$

for some positive constant $A$. The desired result now follows by Theorem $5^{*}$.

\section{REFERENCES}

1. D. Borwein, On methods of summability based on integral functions II. Proc. Camb. Phil. Soc., 56 (1960), 125-131.

2. D. Borwein, Relations between Borel-type methods of summability, Journal London Math. Soc., 35 (1960), 65-70.

3. D. Borwein and B. L. R. Shawyer, On Borel-type methods, Tôhoku Math. Journ., 18 (1966), 283-298.

4. D. Borwein and B. L. R. Shawyer, On Borel-type methods II, Tôhoku Math. Journ., 19 (1967), 232-237.

5. D. Borwein and E. Smet, Tauberian theorems for Borel-type methods of summability, Canad. Math. Bull., 17 (1974), 167-173.

6. D. Gaier, Über die Äquivalenz der $\left|B_{k}\right|$-Verfahren. Math. Z., 64 (1956), 183-191.

7. G. H. Hardy, Divergent Series, Oxford (1949).

8. B. L. R. Shawyer, On the relations between the Abel and Borel-type methods of summability, Proc. Amer. Math. Soc., 22 (1969), 15-19. 
9. B. L. R. Shawyer, Some relations between strong and ordinary Borel-type methods of summability, Math. Z., 109 (1969), 115-120.

DepartMent of Mathematics,

THE UNIVERSITY OF WeSTERn ONTARIO,

LONDON, ONTARIO,

CANADA N6A 5B9 